RESEARCH ARTICLE

\title{
Intratumoral administration of an adenovirus expressing a kinase dead form of ErbB-2 inhibits tumor growth
}

\author{
K Palmer, N Sharan, P Emtage, J Gauldie, WJ Muller and Y Wan \\ Centre for Gene Therapeutics, Department of Pathology and Molecular Medicine, McMaster University, Hamilton, Ontario, Canada
}

\begin{abstract}
ErbB-2 is amplified or overexpressed in a number of different cancers including breast, ovarian, lung, prostate and stomach. This overexpression leads to enhanced receptor dimer formation and stabilization allowing the receptor to remain in an active state. The clinical consequences of ErbB-2 overexpression include increased tumor aggressiveness, poor prognosis, decreased patient survival and resistance to chemotherapy. As a result, a variety of different strategies are being examined to inhibit its function or expression. In this study, we explored the efficacy of a type 5 recombinant adenovirus encoding a kinase dead form of ErbB-2, AderbB-
\end{abstract}

$2 \Delta t k$, as a potential therapeutic agent for breast cancer using a murine breast model expressing constitutively active ErbB2. Co-expression in tumor cells of the kinase dead form of ErbB-2 inhibits receptor activity and induces the death of cells expressing constitutively active ErbB-2. In addition, AderbB-2Atk exhibits antitumor activity in both immune-competent and immune-deficient animals with increased antitumor activity in the immune-competent animals. The results suggest both immune and non-immune mechanisms contribute to the antitumor efficacy of this vector.

Gene Therapy (2002) 9, 898-905. doi:10.1038/sj.gt.3301712

Keywords: ErbB-2; dominant negative; adenovirus; gene therapy; breast cancer

\section{Introduction}

The oncogene erbB-2, a $185 \mathrm{kDa}$ transmembrane tyrosine kinase, is a member of the epidermal growth factor receptor family. ${ }^{1,2}$ ErbB-2 is amplified or overexpressed in a number of different cancers including breast, ovarian, lung, prostate and stomach. ${ }^{3-6}$ This overexpression leads to enhanced receptor dimer formation and stabilization allowing the receptor to remain in an active state. ${ }^{7}$ The clinical consequences of ErbB-2 overexpression include increased tumor aggressiveness, poor prognosis, decreased patient survival and resistance to chemotherapy. ${ }^{3,8-11}$ In transgenic animal models, expression of constitutively active ErbB-2 or overexpression of wildtype ErbB-2 under the control of the mouse mammary tumor virus (mmtv) promoter leads to development of mammary carcinomas. ${ }^{12-14}$ In addition, increased ErbB-2 expression and activation enhances the malignant phenotype and metastatic potential of cancer cells both in vitro and in vivo. ${ }^{15,16}$

These results suggest ErbB-2 overexpression and activation play an important role in the development of cancer. Therefore inhibition of ErbB-2 function may be an effective antitumor therapy. A number of different strategies are being developed to inhibit ErbB-2 function or expression. These strategies include the use of tyrosine kinase inhibitors, adenoviral E1A protein, anti-ErbB-2

Correspondence: Y Wan, Health Sciences Centre, Room 4H21B, Department of Pathology and Molecular Medicine, McMaster University, 1200 Main Street West, Hamilton, Ontario L8N 3Z5, Canada

Received 21 September 2001; accepted 11 February 2002 antibodies, antisense constructs and ribozymes targeting ErbB-2. Inhibition of ErbB-2 tyrosine kinase activity in cancer cells overexpressing ErbB-2 leads to suppression of tumor growth and prolonged mouse survival. ${ }^{17-19}$ In addition, repression of ErbB-2 activity sensitizes previously resistant ErbB-2 overexpressing lung cancer cells to a variety of chemotherapeutics. ${ }^{20}$

ErbB-2 receptor signaling involves ligand binding, homo- and heterodimerization with other ErbB family members, such as ErbB-3, leading to kinase activation, trans-tyrosine phosphorylation and signal transduction. ${ }^{21,22}$ When kinase dead forms of ErbB-2 are coexpressed with ErbB-2, the kinase dead ErbB-2 acts in a dominant negative fashion. Dimer formation between the two leads to receptor inactivation. Previously, Messerle et $a l^{23}$ demonstrated expression of kinase domain mutants in transformed NIH 3T3 (ErbB-2 positive) cells causes a reversion of the transformed phenotype. Therefore, overexpression of a kinase dead mutant form of ErbB-2 using an adenoviral construct (AderbB-2 $\Delta$ tk) may block tyrosine kinase activity and inhibit signaling which may inhibit cell proliferation or induce apoptosis.

In addition to inhibiting the signaling of endogenous ErbB-2, intratumoral injection of AderbB-2 $\Delta$ tk may stimulate an antitumor immune response. ErbB-2 has been previously identified as a target for tumor immunotherapy. Pre-existent humoral and cellular immune responses directed against ErbB-2 are observed in breast cancer patients, although not at the levels necessary to prevent tumor formation. ${ }^{24,25}$ The existence of ErbB-2 immunity in cancer patients suggests appropriately constructed vaccines may be able to induce or stimulate an 
antitumor immune response. Augmentation of this immune response through genetic immunization with plasmids expressing ErbB-2 has been demonstrated in animal models. ${ }^{26}$ Amici et al ${ }^{26}$ generated a strong $\mathrm{T}_{\mathrm{H}} 1$ immune response against ErbB-2 following intramuscular injection of a plasmid expressing ErbB-2. This immune response inhibited tumor formation in transgenic mice overexpressing ErbB-2. In addition, immunization of mice with a vaccinia virus expressing the extracellular domain of ErbB-2 induced immune responses against ErbB-2 that protected against subsequent tumor challenge. ${ }^{27}$ Recently, we have demonstrated that dendritic cells transduced ex vivo with AderbB-2 $\Delta$ tk induced both protective and therapeutic immunity against tumor cells expressing ErbB-2. ${ }^{28}$

In this study, we investigated the antitumor effects of direct intratumoral administration of a recombinant adenovirus vector expressing a kinase dead form of ErbB2 (AderbB-2 $\Delta$ tk). Expression of the kinase dead ErbB-2 down-regulates the activity of endogenous ErbB-2 and induces apoptosis both in vitro and in vivo. However, complete tumor protection seems to require the development of an anti-ErbB-2 immune response because a greater antitumor response was observed in immunecompetent animals. This is supported by the increase of infiltrated $\mathrm{CD}^{+} \mathrm{T}$ cells in the tumor and CTL response. The results demonstrate that both immune and nonimmune mechanisms play a role in the antitumor effects exhibited by AderbB-2 $\Delta$ tk.

\section{Results}

Effect of adenoviral infection on NDL cells in vitro We have previously demonstrated that infection of ErbB2-negative cells with AderbB-2 $\Delta$ tk resulted in overexpression of ErbB-2 $\Delta$ tk protein determined by FACS analysis and immunohistochemical staining. ${ }^{28}$ To test whether AderbB-2 $\Delta$ tk infection has any impact on the growth of ErbB-2-positive cells due to overexpression of the kinase dead form of ErbB-2, NDL cells (which express constitutively active ErbB-2) were infected at a MOI of 100 with either control vector Ad5 BHG $\Delta \mathrm{E} 1 / \Delta \mathrm{E} 3$, or AderbB-2 $\Delta$ tk. As controls, normal mouse skin fibroblasts and B16F10 melanoma cells (which do not express active ErbB-2) were also infected with the same vectors. Five days after infection, cells were trypsinized, stained with trypan blue and counted. Large numbers of dead cells were observed in the AderbB-2 $\Delta$ tk-infected NDL cell dishes as compared with control virus infection (approximately 30-fold increase in dead cells in the AderbB-2 $\Delta$ tk-treated dishes as compared with control adenovirus treated dishes) (Figure 1). AderbB-2 $\Delta$ tk infection did not induce cell death and had no effect on the growth of normal mouse skin fibroblasts and B16F10 in vitro (data not shown). These results suggest that the overexpression of a kinase dead form of ErbB-2 by AderbB-2 $\Delta$ tk specifically kills cells expressing activated ErbB-2.

\section{AderbB-2 $\Delta$ tk inhibited ErbB-2 mediated transformation of Rat-1 fibroblasts}

To assess the effect of kinase dead ErbB-2 expression on ErbB-2-mediated transformation, Rat-1 fibroblasts transfected with plasmids expressing activated ErbB-2, poly-

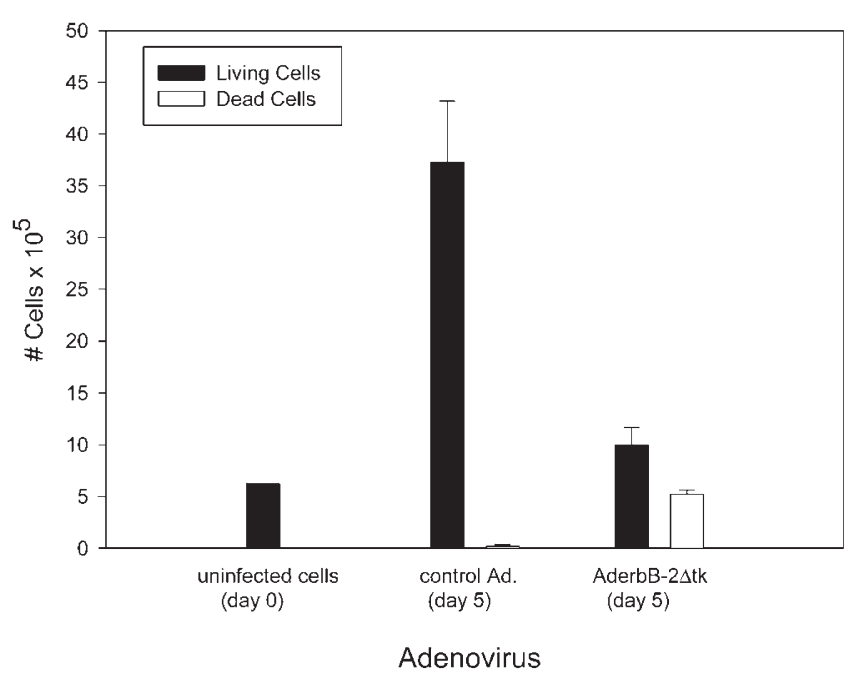

Figure 1 Effect of adenoviral infection on NDL cell growth in vitro. To test the growth inhibitory effects of AderbB-2 $\Delta$ tk in vitro, NDL cells were infected at a multiplicity of infection (MOI) of 100 with either control adenovirus or AderbB-2 $\Delta t k, 5$ days after infection cells were trypsinized and counted. Cell viability was determined using trypan blue.

oma middle $\mathrm{T}$ or activated Ras were infected with AderbB-2 $\Delta$ tk or control adenovirus. AderbB-2 $\Delta$ tk infection dramatically inhibits the transforming activity of activated ErbB-2 (no foci observed in AderbB-2 $\Delta$ tk as compared with $158 \pm 15$ foci observed in control plates). This inhibition of transformation following AderbB-2 $\Delta$ tk infection was specific for ErbB-2, as AderbB-2 $\Delta$ tk infection had no effect on the transforming activity of polyoma middle $\mathrm{T}$ or activated Ras (Table 1 ). In addition, control virus infection did not inhibit transforming activity suggesting the suppression of focus forming activity is not a viral effect $(161 \pm 22$ foci in control adenovirusinfected plates as compared with $158 \pm 15$ foci in uninfected plates). These results suggest that the expression of a kinase dead ErbB-2 by AderbB-2 $\Delta$ tk specifically inhibits the activity of ErbB-2.

To further examine the mechanism by which AderbB$2 \Delta$ tk inhibits the transforming activity of ErbB-2 we examined ErbB-2 expression and phosphorylation levels in AderbB-2 $\Delta$ tk infected and control Rat-1 fibroblasts expressing activated ErbB-2. While AderbB-2 $\Delta$ tk infection did not down-regulate expression of total ErbB-2

Table 1 Effect of AderbB-2 $\Delta$ tk on transformation of Rat-1 fibroblasts transfected with either activated ErbB-2 (NeuNT), middle T antigen or activated Ras (RasV12)

\begin{tabular}{|c|c|}
\hline Construct & $\begin{array}{c}\text { Focus assay (average } \\
\text { No. of foci) }\end{array}$ \\
\hline NeuNT & $158 \pm 15$ \\
\hline NeuNT + control adenovirus & $161 \pm 22$ \\
\hline NeuNT + AderbB-2 $\Delta$ tk & 0 \\
\hline Middle $\mathrm{T}$ antigen & $214 \pm 27$ \\
\hline Middle $T$ antigen + AderbB-2 $\Delta$ tk & $216 \pm 13$ \\
\hline RasV12 & $184 \pm 21$ \\
\hline RasV12 + AderbB-2 $\Delta$ tk & $188 \pm 18$ \\
\hline
\end{tabular}

aValues for each assay represent mean number of foci/plate counted on three plates \pm standard deviation. This experiment was repeated three times with similar results. 
protein levels (Figure 2a), a reduction in tyrosine phosphorylation of ErbB-2 was observed in AderbB-2 $\Delta$ tk infected cells (Figure 2b). In addition, control adenovirus infection did not inhibit tyrosine phosphorylation of ErbB-2, suggesting that overexpression of a kinase dead mutant form of ErbB-2 using an adenoviral construct may inhibit ErbB-2 signaling pathway by downregulating its tyrosine kinase activity.

\section{Determination of apoptosis in adenoviral treated NDL tumors}

The fact that AderbB-2 $\Delta$ tk infection results in the death of NDL cells in vitro lead us to investigate whether intratumoral injection with this vector can induce tumor cell death in vivo. To determine this, naive FVB or SCID Beige mice were injected s.c. with $7 \times 10^{6} \mathrm{NDL}$ cells allowing the development of palpable tumors in 21 days. Intratumoral injection was performed with $10^{9}$ p.f.u. of either control adenovirus or AderbB-2 $\Delta$ tk and tumor tissues were examined for the presence of apoptotic cells using the Apoptag In Situ Apoptosis Detection Kit. Dramatic increases in apoptotic cells were observed in AderbB$2 \Delta$ tk treated SCID and FVB mice as compared with control virus treated tumors at both day 1 and day 5 after intratumoral injection (Figure 3 and data not shown). These results, in addition to the in vitro data demonstrating that AderbB-2 $\Delta$ tk infection results in a specific inhibition of ErbB-2 activity and NDL cell death, suggest

a
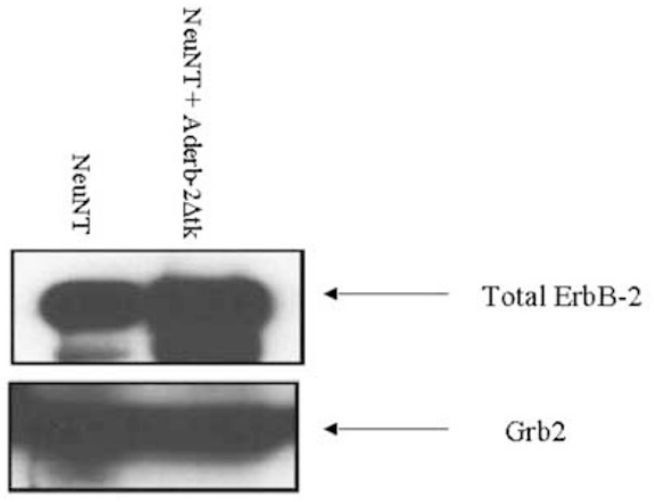

Grb2
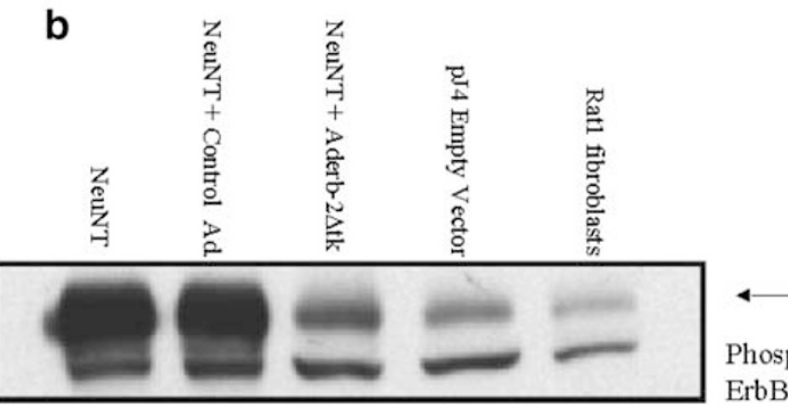

PhosphErbB-2

Figure 2 AderbB-2 $\Delta$ tk infection specifically reduces tyrosine phosphorylation of ErbB-2. To determine the effect of AderbB-2 $\Delta t k$ infection on ErbB2 expression and tyrosine phosphorylation, protein lysates from Rat-1 fibroblasts electroporated with $\mathrm{pJ} 4 \Omega$-derived plasmids (NeuNT (activated ErbB-2) or control plasmid) and infected with AderbB-2Atk or control adenovirus were subjected to Western blot analysis with anti-ErbB-2 antibody or anti-Grb-2 (control for equal loading) (a) or immunoprecipitated with anti-phosphotyrosine antibody (PY20) and then subjected to an immunoblot analysis with anti-ErbB-2 (b).
AderbB-2 $\Delta$ tk infection may have a therapeutic effect on tumor growth by inducing apoptosis of cancer cells expressing activated ErbB-2.

\section{Antitumor efficacy of AderbB-2stk}

To examine the therapeutic benefit obtained by injecting AderbB-2 $\Delta$ tk vector into ErbB-2 positive tumors in vivo, virus-injected tumor bearing FVB or SCID Beige mice were observed for 2 months. Intratumoral administration of the control vector, Ad5 BHG $\Delta \mathrm{E} 1 / \Delta \mathrm{E} 3$, did not prevent NDL tumor progression in either immune-competent FVB or immune-deficient SCID beige mice, while injection of AderbB-2 $\Delta$ tk resulted in significant tumor regression and growth delay in $100 \%$ of FVB mice treated. Importantly, 52\% of AderbB-2 $\Delta$ tk-treated FVB mice exhibited complete tumor regression for more than 8 weeks (Figure 4a). In contrast, although intratumoral injection of AderbB-2 $\Delta$ tk resulted in partial tumor regression or growth delay in $100 \%$ of immune-deficient animals treated (Figure 4b), all animals succumbed to eventual tumor growth. These data indicate that although overexpression of a kinase dead ErbB-2 could induce apoptosis in NDL tumors leading to initial inhibition of tumor growth, inhibition of ErbB-2 function alone is not sufficient to induce complete tumor regression. Rather the fact that complete regressions only occurred in immune-competent FVB mice suggests that subsequent immune responses may be required to completely eliminate tumors.

Since adenoviral vectors expressing tumor antigen have been suggested as a cancer vaccine that is able to stimulate antitumor immune responses and induce tumor regression, we have also included a group of tumor-bearing FVB mice where AderbB-2 $\Delta$ tk virus was injected s.c. outside of tumors. Interestingly, only intratumoral but not s.c. injection with AderbB-2 $\Delta$ tk vector could induce complete tumor regression suggesting that intratumoral injection-induced initial inhibition of tumor

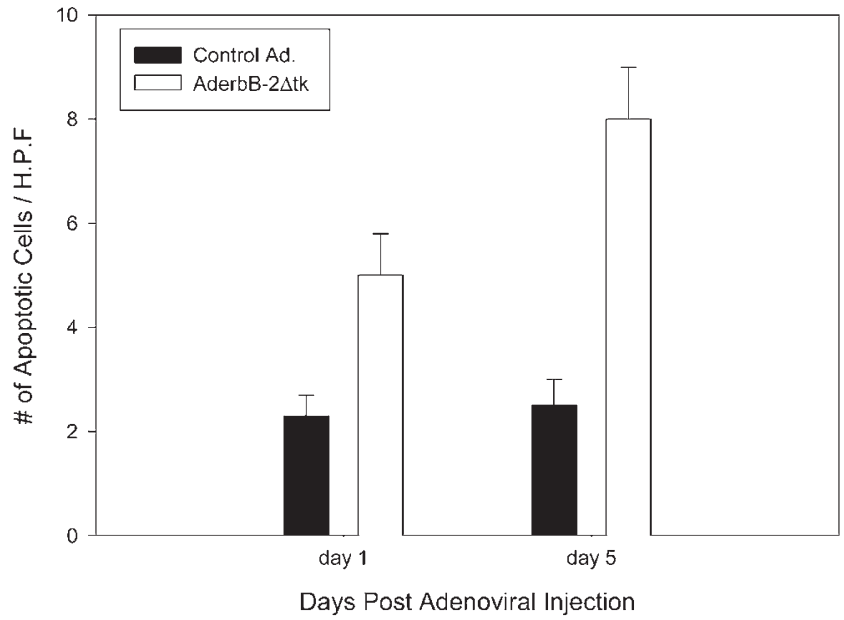

Figure 3 Determination of apoptosis in treated NDL tumors. Apoptag In Situ Apoptosis Detection Kit-Peroxidase was used to examine apoptosis in AderbB-2 $\Delta$ tk and control adenovirus treated NDL tumors from SCID beige mice. One and 5 days after adenoviral administration, tumors were removed, fixed in $10 \%$ buffered formalin and paraffin embedded. Sections (3-5 $\mu \mathrm{m})$ were processed and subjected to Apoptag. To determine relative numbers of apoptotic cells in tumors five high power fields (HPF) were randomly selected per section and positively staining cells were counted and averaged. 

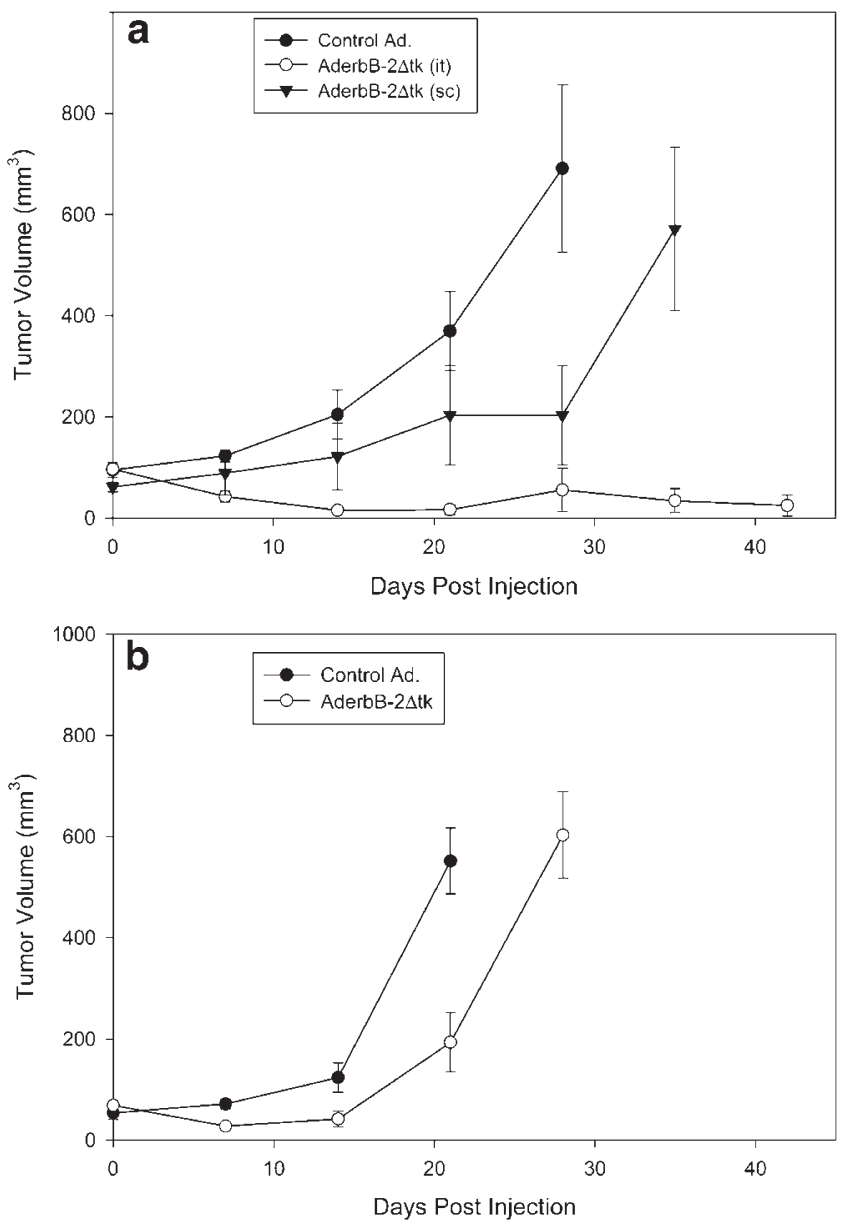

Figure 4 Effect of direct intratumoral administration of recombinant adenoviruses on NDL tumor growth in immune-competent FVB mice and immune-deficient SCID beige mice. Following palpable tumor formation, mice (a, immune-competent FVB mice; $b$, immune-deficient SCID beige mice) were intratumorally or subcutaneously (opposite flank) injected with $10^{9}$ p.f.u. control virus or AderbB-2 $\Delta$ tk. Tumor volume was determined by measuring the length, width and depth of the tumor weekly using calipers. Animals were killed when any two measurements were greater than $20 \mathrm{~mm}(n=10)$.

growth and induction of apoptosis were critical for development of immune responses (Figure 4a). In addition, to characterize the specificity of AderbB-2 $\Delta$ tk, we examined the ability of direct intratumoral injection of AderbB-2 $\Delta$ tk to inhibit the growth of ErB-2-negative tumors. We failed to detect any inhibition of ErB-2-negative B16F10 melanoma tumor growth in C57BL/ 6 mice by intratumoral injection of AderbB-2 $\Delta$ tk indicating that therapeutic effect of AderbB-2 $\Delta$ tk is limited to tumors expressing ErbB family members (data not shown).

\section{Examination of antitumor immune response in FVB mice} following AderbB-2stk treatment

The greater antitumor efficacy of AderbB-2 $\Delta$ tk observed in immune-competent animals suggests that both immune and non-immune mechanisms may be involved. To further characterize the antitumor activity of AderbB$2 \Delta$ tk in vivo, we examined tumors from FVB mice 1 and 5 days after adenoviral injection for accumulation of lymphocytes using anti-CD3 immunohistochemistry. In control vector-treated tumors limited lymphocyte infiltration
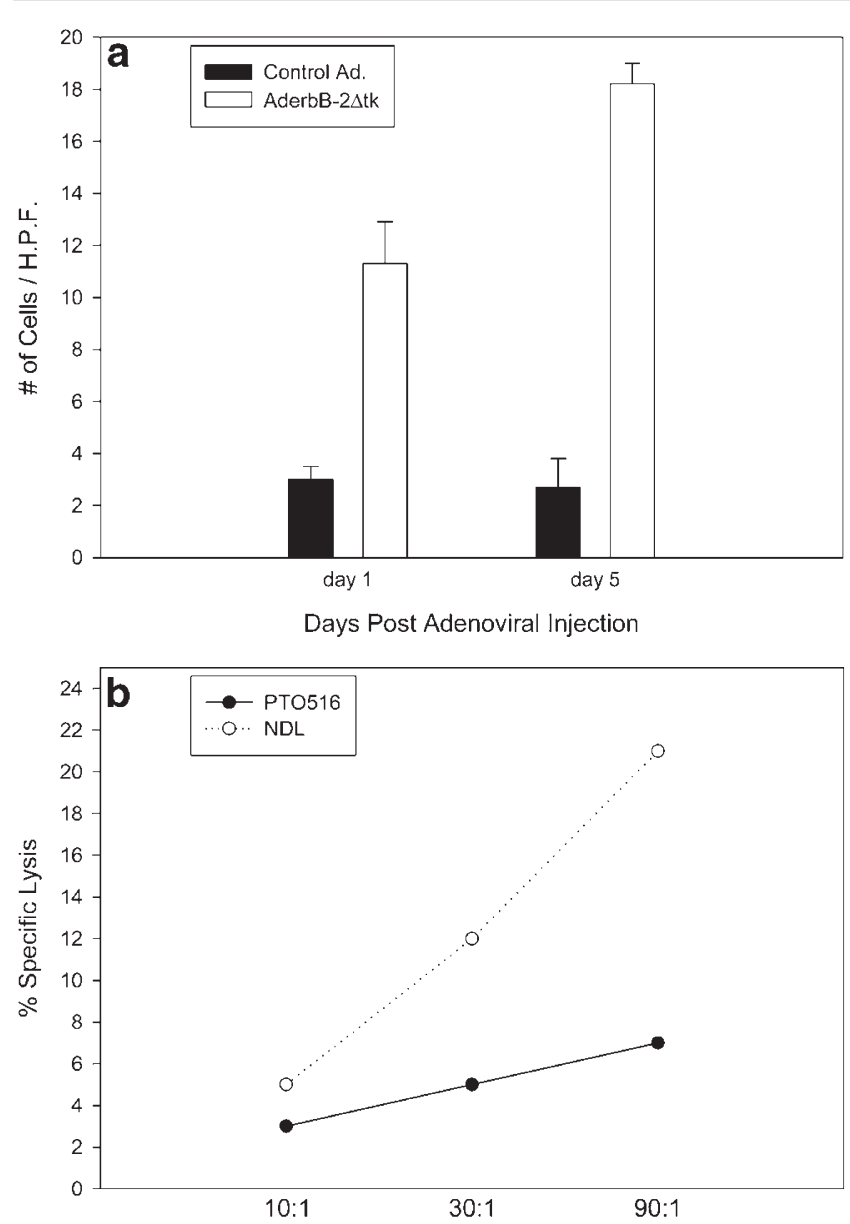

Figure 5 Examination of antitumor immune response in NDL tumors bearing FVB mice following AderbB-2 $\Delta$ tk treatment. Immunohistochemistry was used to examine the $\mathrm{CD}^{+}$cell infiltrate in adenoviral vector treated tumors. One and 5 days after adenoviral administration tumors were removed, fixed in $10 \%$ buffered formalin and paraffin embedded. Sections (3-5 $\mu \mathrm{m})$ were processed and subjected to anti-CD3 immunohistochemistry. To determine the relative numbers of $\mathrm{CD}^{+}$cells in tumors five high power fields (HPF) were randomly selected per section and positively staining cells were counted and averaged (a). To determine if tumor regression induced by intratumoral injection of AderbB-2Atk is associated with the presence of tumor antigen-specific CTL, spleens were removed from AderbB-2 $\Delta$ tk-treated NDL tumor bearing mice which had undergone complete tumor regression. The splenocytes were isolated and restimulated for 5 days by coculture with irradiated NDL cells. Following coculture, the splenocytes were used in a ${ }^{51} \mathrm{Cr}$ release assay using ${ }^{51} \mathrm{Cr}$ labeled $\mathrm{NDL}$ (ErbB-2 positive) or PTO516 (ErbB-2 negative) as targets.

was observed at day 1 and day 5 after vector administration. In contrast, considerable lymphocyte infiltration was observed at both day 1 and day 5 after AderbB-2 $\Delta$ tk administration (Figure 5a). The results suggest AderbB$2 \Delta$ tk in addition to inhibiting ErbB-2 function stimulates an antitumor immune response. Indeed, this hypothesis is further supported by a tumor specific CTL response from regressed FVB mice (AderbB-2 $\Delta$ tk treated) (Figure $5 b)$.

\section{Discussion}

The erbB-2 oncogene plays a critical role in the development of breast cancers. As a result, a variety of different strategies are being explored to inhibit its function or 
expression. One such strategy is the use of inactive mutant forms of ErbB-2 binding partners to act in a dominant negative fashion and inhibit ErbB-2 activity. ErbB-2 receptor signaling involves homo- and heterodimerization with ErbB family members leading to kinase activation, trans-tyrosine phosphorylation and signal transduction. ${ }^{21,22}$ When inactive forms of ErbB-2 binding partners are co-expressed with ErbB-2, dimer formation between the two leads to receptor inactivation. Previously the expression of inactive ErbB-2 has been demonstrated to inhibit activity of ErbB-2 and reverse ErbB-2 mediated transformation in vitro. ${ }^{23}$

In this study, we explored the efficacy of a type 5 recombinant adenovirus encoding a kinase dead form of ErbB-2 as a potential therapeutic agent for breast cancer using a murine breast model expressing constitutively active ErbB-2. We have demonstrated that intratumoral administration of a vector expressing a kinase dead form of ErbB-2 exhibits antitumor activity in both immunecompetent and immune-deficient animals with increased antitumor activity in the immune-competent animals. The results suggest both immune and non-immune mechanisms contribute to the antitumor efficacy of this vector.

The non-immune mechanisms underlying AderbB$2 \Delta$ tk antitumor activity may include the induction of apoptosis due to decreased receptor activity. Previously, Deshane et $a l^{29,30}$ demonstrated that inhibition of ErbB-2 expression by administration of an adenovirus expressing $\mathrm{sFv}$ directed against ErbB-2 induces apoptosis of ErbB-2 overexpressing tumor cells. We observed similar results with AderbB-2 $\Delta$ tk. AderbB-2 $\Delta$ tk infection in vitro dramatically induces death of cells expressing activated ErbB-2 as compared with control adenovirus-treated cells (Figure 1). In addition, increased levels of apoptosis were observed in NDL tumors treated with AderbB-2 $\Delta$ tk (Figure 3). Therefore inhibition of ErbB-2 receptor signaling by co-expression of a kinase dead form ErbB-2 induces apoptosis of cancer cells. While induction of apoptosis in NDL tumors had antitumor activity (Figure $4 \mathrm{~b})$, it was not sufficient to induce complete tumor regressions. Possibly the transient nature of adenoviral infection and the inability to infect all tumor cells lead to this inability to induce complete tumor regressions.

In addition to inducing apoptosis of cells over-expressing ErbB-2 or expressing activated ErbB-2, AderbB-2 $\Delta$ tk may induce reversion of the transformed phenotype. Previously, Messerle et $a^{23}$ have demonstrated that cells transformed with an activated erbB-2 oncogene can be reverted by expression of a kinase deficient ErbB-2. Inhibition of ErbB-2 function by PEA3 has also been shown to suppress cell growth and transformation. ${ }^{31}$ Infection of Rat-1 fibroblasts expressing activated ErbB-2, polyoma middle $\mathrm{T}$ or activated Ras with AderbB- $2 \Delta$ tk specifically suppressed the transforming activity induced by ErbB-2 and had no effect on polyoma middle $\mathrm{T}$ or Ras mediated transformation (Table 1). This inhibition of ErbB-2mediated transformation by AderbB-2 $\Delta$ tk resulted from the inhibition of ErbB-2 tyrosine phosphorylation not ErbB-2 expression (Figure 2).

In addition to inhibiting the signaling of endogenous ErbB-2, AderbB-2 $\Delta$ tk may stimulate an anti-ErbB-2 immune response. ErbB-2 has been identified as a tumor antigen and pre-existent anti-ErbB-2 immune responses are observed in some cancer patients. ${ }^{24}$ Therefore appro- priately constructed vaccines may be able to augment an anti-ErbB-2 immune response. Stimulation of this immune response following immunization with ErbB-2 expression vectors has been observed in animal models. Amici et $a l^{26}$ generated a strong anti-ErbB-2 Th1 immune following intramuscular injection of a plasmid expressing ErbB-2 that inhibited tumor formation in transgenic mice overexpressing ErbB-2. In addition, immunization with a vaccinia virus expressing the extracellular domain of ErbB-2 induced both cellular and humoral immune responses against ErbB-2 that protected against subsequent tumor challenge. ${ }^{27}$ Recently, we have shown dendritic cells infected with AderbB-2 $\Delta$ tk could induce protective immunity against NDL tumor cell challenge and dramatically suppressed the growth of pre-existing tumors. ${ }^{28}$

Enhanced AderbB-2 $\Delta$ tk antitumor efficacy was observed in immune-competent FVB mice as compared with immune-deficient SCID Beige mice (Figure 4). These results suggest that intratumor administration of AderbB$2 \Delta$ tk stimulates an immune response against ErbB-2 in addition to inhibiting ErbB-2 receptor function. In support of this, we are able to demonstrate the presence of anti-ErbB-2 circulating CTL in animals treated with AderbB-2 $\Delta$ tk. In addition, an accumulation of lymphocytes in AderbB-2 $\Delta$ tk-treated tumors was observed 1 and 5 days after AderbB-2 $\Delta$ tk infection (Figure 5). While intratumor injection of AderbB-2 $\Delta$ tk could induce tumor regression, extratumoral injection with this same vector did not. A number of groups have demonstrated genetic vaccination with ErbB-2 in non-tumor-bearing animals leads to the development of protective immunity. ${ }^{26}$ Possibly AderbB-2 $\Delta$ tk vaccination is less efficient in tumorbearing animals. Alternatively, the apoptosis of tumor cells following intratumoral administration of AderbB$2 \Delta$ tk provides tumor antigens to dendritic cells through apoptotic bodies and stimulates an antitumor immune response. In support, Ronchetti et al ${ }^{32}$ demonstrate induction of an antitumor immune response following injection of apoptotic tumor cells. In addition, Shi et al ${ }^{33}$ demonstrate the presence of endogenous adjuvant activity in cells that stimulate CTL response following release from the cell. This endogenous adjuvant activity increases as the cell undergoes apoptosis.

The studies reported here demonstrate the therapeutic efficacy of an adenovirus expressing kinase dead ErbB-2 against murine breast cancer cells in vivo. In our model, AderbB-2 $\Delta$ tk inhibited tumor growth in both immunecompetent and immune-deficient animals. However the increased antitumor efficacy observed in immune-competent animals suggests both immune and non-immune mechanisms contribute to the antitumor activity of AderbB-2 $\Delta$ tk. Possibly the therapeutic effect AderbB$2 \Delta$ tk could be enhanced by the addition of immune stimulatory molecules or other dominant negative ErbB family members.

\section{Materials and methods}

\section{Animals}

Six- to 8-week-old FVB, C57BL/6, and Balb/c SCID beige female mice were purchased from Charles River Laboratories (Wilmington, MA, USA) and housed in pathogenfree facility until use. Animal studies were conducted 
according to regulations of the Canadian Council on Animal Care and approved by the Animal Research Ethics Board at McMaster University, Canada.

\section{Recombinant adenoviruses}

The vector expressing a kinase dead form of rat ErbB-2, AderbB-2 $\Delta \mathrm{tk}$, was constructed as previously described. ${ }^{28}$ The vector was shown to express protein by FACS analysis of cells infected with either control virus or AderbB$2 \Delta$ tk for $24 \mathrm{~h}$ and then labeled with anti-ErbB-2/neu $\mathrm{mAb}{ }^{28}$ The control vector, Ad5 BHG $\Delta \mathrm{E} 1 / \Delta \mathrm{E} 3$, is an E1 and E3 deleted type 5 adenovirus with no inserted foreign genes. ${ }^{34}$ Using a previously described biological assay for replication competent adenovirus (RCA), AderbB-2 $\Delta$ tk and Ad5 BHG $\Delta \mathrm{E} 1 / \Delta \mathrm{E} 3$ were determined to be RCA negative. ${ }^{35}$

\section{Cell lines and tumor induction}

The murine breast cancer model was developed from tumors arising in transgenic mice (NDL 8142) expressing a constitutively active mutated form of ErbB-2 in the mammary epithelium. ${ }^{36,37}$ A cell line (NDL) generated from tumors isolated from the breast epithelium of these transgenic mice was used to establish tumors in syngenic recipient female mice (FVB) or Balb/c SCID beige female mice. Briefly, a single cell suspension of NDL cells was washed with phosphate buffered saline (PBS), and $7 \times$ $10^{6}$ cells were injected subcutaneously into the right hind flank. Palpable tumors (75 to $150 \mathrm{~mm}^{3}$ ) developed within 21 days.

Rat-1 fibroblasts used in this study were obtained from the American Tissue Type Collection (ATCC) (Rockville, MD, USA). They were maintained in Dulbecco's modified Eagle medium (DMEM) supplemented with $10 \%$ fetal bovine serum (FBS), streptomycin (100 $\mu \mathrm{g} / \mathrm{ml})$, penicillin $(100 \mathrm{U} / \mathrm{ml})$, and fungizone $(1 \mu \mathrm{g} / \mathrm{ml})$ (GIBCO BRL, Burlington, Ontario, Canada).

Normal mouse skin fibroblasts were generated from the skin of C57BL/ 6 mice. B16F10 melanoma cell line was obtained from National Cancer Institute (Bethesda, MD, USA). These cells were cultured in RPMI supplemented with $10 \%$ fetal bovine serum (FBS), streptomycin (100 $\mu \mathrm{g} / \mathrm{ml}$ ) and penicillin (100 U/ml) (GIBCO BRL). For tumor induction, $1 \times 10^{4}$ B16 F10 cells were injected subcutaneously into the right hind flank of syngeneic recipients (female C57BL/6 mice). B16 F10 tumors were allowed to reach a palpable size $\left(75\right.$ to $150 \mathrm{~mm}^{3}$ ) before adenoviral injection.

\section{Effect of adenoviral infection on NDL cells in vitro}

To test the growth inhibitory effects of AderbB-2 $\Delta$ tk in vitro, NDL cells (express constitutively active ErbB-2) or normal mouse skin fibroblasts were infected at a multiplicity of infection (MOI) of 100 with either control adenovirus or AderbB-2 $\Delta$ tk, 5 days after infection cells were trypsinized and counted. Cell viability was determined using trypan blue.

\section{Focus-forming assay}

To determine if infection with AderbB-2 $\Delta$ tk inhibited ErbB-2 transformation of Rat-1 fibroblasts, a focus-forming assay was performed as previously described with the following modifications. ${ }^{36}$ Briefly, Rat- 1 fibroblasts (1 $\times 10^{6}$ ) were electroporated with $5 \mu \mathrm{g}$ of $\mathrm{pJ} 4 \Omega$-derived plasmid DNA encoding NeuNT (activated ErbB-2), poly- omavirus middle $\mathrm{T}$ antigen, or Rasv12, and $0.1 \mu \mathrm{g}$ of a puromycin resistence plasmid (PGKpuro). Eletroporations were performed with a BioRad (Missasauga, Ontario, Canada) gene pulser set at $1000 \mathrm{~V}$ and $25 \mu \mathrm{F}$. The cells were plated at a density of $10^{6}$ cells per $100 \mathrm{~mm}$ tissue culture dish. After $24 \mathrm{~h}$, the cells were infected with adenovirus (control or AderbB-2 $\Delta$ tk) at a MOI of 70 and were maintained in a monolayer (using DMEM containing $2 \%$ FBS) for a total of 14 days. The plates were fixed with $10 \%$ buffered formalin phosphate and stained with Giemsa stain (Fisher Scientific, Nepean, Ontario, Canada). One plate per construct was kept for protein analysis. The number of foci on each of the stained plates was counted.

\section{Protein extraction, immunoprecipitation and immune blotting}

One plate per construct from the focus-forming assay was used for protein analysis. Cells were then washed twice with ice-cold $1 \times$ PBS and lysed for $20 \mathrm{~min}$ in TNE lysis buffer (50 mM Tris- $\mathrm{HCl}$ (pH 7.6), $150 \mathrm{mM} \mathrm{NaCl}, 1 \%$ Nonidet P-40, 2 mM EDTA, $1 \mathrm{mM}$ sodium orthovanadate, $2 \mathrm{mM}$ dithiothreitol, $10 \mu \mathrm{g}$ of leupeptin per $\mathrm{ml}, 10$ $\mu \mathrm{g}$ of aprotinin per $\mathrm{ml}$ ). The lysates were cleared by centrifugation at $12000 \mathrm{~g}$ for $10 \mathrm{~min}$ at $4^{\circ} \mathrm{C}$ and protein concentrations were determined by Bradford assay (BioRad).

To determine total ErbB-2 levels, the lysates were electrophoresed on 4-12\% gradient SDS polyacrylamide gels and transferred on to polyvinylidene difluoride (PVDF) membranes (Immobilon-P; Millipore, Etobicoke, Ontario, Canada) with an immunoblot transfer apparatus (BioRad). The membranes were probed with anti-ErbB-2 (Ab.3, Oncogene Science, Cambridge, MA, USA) antibody or anti-Grb2 rabbit polyclonal sera (C23, Santa Cruz Biotechnology, Santa Cruz, CA, USA) (control for equal loading).

Immunoprecipitations were performed by incubating $600 \mu \mathrm{g}$ of the protein lysate with anti-phosphotyrosine antibody (PY20) and protein G-sepharose beads (Amersham Pharmacia Biotech, Baie D'urse, Quebec, Canada) for $3 \mathrm{~h}$ at $4^{\circ} \mathrm{C}$. The immunoprecipitates were then washed five times in TNE lysis buffer and resuspended in SDS gel loading buffer $(62.5 \mathrm{mM}$ Tris- $\mathrm{HCl}(\mathrm{pH}$ 6.8), 2\% SDS, 5\% glycerol, $0.8 \mathrm{M}$ 2-mercaptoethanol, $0.25 \%$ bromophenol blue), electrophoresed on $4-12 \%$ gradient SDS polyacrylamide gels and transferred on to PVDF membranes. The membranes were probed with anti-ErbB-2 antibody. Proteins were visualized using an enhanced chemiluminesence detection system (Amersham Pharmacia Biotech).

\section{Intratumor vector administration}

Following palpable tumor formation (approximately 75$150 \mathrm{~mm}^{3}$ ) the animals were injected intratumorally with $50 \mu \mathrm{l}$ of $10^{9}$ p.f.u. recombinant adenoviruses (Ad5 BHG $\Delta \mathrm{E} 1 / \Delta \mathrm{E} 3$ or AderbB-2 $\Delta \mathrm{tk}$ ) diluted in sterile endotoxinfree phosphate buffered saline (PBS). Tumor volume was determined by measuring the length, width and depth of the tumor weekly using calipers. Animals were killed when any two measurements were greater than $20 \mathrm{~mm}$.

\section{Determination of apoptosis in treated NDL tumors}

Apoptag In Situ Apoptosis Detection Kit-Peroxidase (Intergen, Purchase, NY, USA) was used to examine apoptosis in adenovirally treated NDL tumors from SCID 
beige and FVB mice. Tumors were removed 1 and 5 days after administration of various vectors. All tumors were fixed in $10 \%$ buffered formalin and paraffin embedded. Sections (3-5 $\mu \mathrm{m})$ were processed and subjected to Apoptag. To determine the relative numbers of apoptotic cells in tumors five high power fields (HPF) were randomly selected per section and positively staining cells were counted and averaged.

\section{Cytotoxic $T$ cell assays}

Splenocytes (effectors) were obtained from mice whose NDL tumors had regressed as a result of adenoviral vector administration. Effectors were co-cultured with irradiated NDL cells (stimulators) at a concentration of $30 \times 10^{6}$ effectors to $0.5 \times 10^{6}$ stimulators for 5 days in six-well plates. Serial dilutions of the effector cells were incubated in a V-bottom 96-well plate with $5 \times 10^{3} \mathrm{NDL}$ or PTO516 (an erbB-2 negative syngenic cell line) target cells. Target cells $\left(10^{6}\right)$ were labeled with $500 \mu \mathrm{Ci}$ of ${ }^{51} \mathrm{Cr}$ $\left(\mathrm{Na}_{2}{ }^{51} \mathrm{CrO}_{4} ; \mathrm{Mandel} / \mathrm{NEN}\right.$ Life Science, Guelph, Ontario, Canada) in $1 \mathrm{ml}$ of medium for 90 min before co-culture with the effector cells. Cells were co-cultured for $4 \mathrm{~h}$, at which time $80 \mu \mathrm{l}$ of supernatant was removed for $\gamma$ counting. The percent specific lysis was calculated as follows: (experimental c.p.m. - spontaneous c.p.m.)/(maximal c.p.m. - spontaneous c.p.m.) $\times 100 \%$.

\section{Determination of $\mathrm{CD}^{+}$cell infiltration in treated $\mathrm{NDL}$ tumors}

Immunohistochemistry was used to examine the $\mathrm{CD}^{+}$ cell infiltrate in adenoviral vector-treated tumors from FVB mice. Tumors were removed 1 and 5 days after administration of various vectors. All tumors were fixed in $10 \%$ buffered formalin and paraffin embedded. Sections $(3-5 \mu \mathrm{m})$ were processed and subjected to anti-CD3 (rabbit anti-human CD3; Dako, Carpinteria, CA, USA) immunohistochemistry. To determine the relative numbers of $\mathrm{CD}^{+}$cells in tumors, five high power fields (HPF) were randomly selected per section and positively staining cells were counted and averaged.

\section{Acknowledgements}

The authors acknowledge the excellent technical help of Duncan Chong, Xueya Feng and Mary Jo Smith. This work was supported by the Canadian Institutes of Health Research, the Breast Cancer Society of Canada, and CANVAC to YW and by the Canadian Breast Cancer Research Initiative to WJM. N Sharan is a recipient of a US Army studentship. WJ Muller is a recipient of CIHR Scientist award.

\section{References}

1 Bargmann CI, Hung MC, Weinberg RA. The neu oncogene encodes an epidermal growth factor receptor-related protein. Nature 1986; 319: 226-230.

2 Yamamoto $\mathrm{T}$ et al. Similarity of protein encoded by the human c-erb-B-2 gene to epidermal growth factor receptor. Nature 1986; 319: $230-234$.

3 Slamon DJ et al. Human breast cancer: correlation of relapse and survival with amplification of the HER-2/neu oncogene. Science 1987; 235: 177-182.

4 Scheurle D et al. HER-2/neu expression in archival non-small cell lung carcinomas using FDA-approved Hercep test. Anticancer Res 2000; 20: 2091-2096.
5 Sadasivan R et al. Overexpression of Her-2/neu may be an indicator of poor prognosis in prostate cancer. J Urol 1993; 150: 126-131.

6 Ishikawa T et al. Amplification of the c-erbB-2 (HER-2/neu) gene in gastric cancer cells. Detection by fluorescence in situ hybridization. Am J Pathol 1997; 151: 761-768.

7 Hung MC, Lau YK. Basic science of HER-2/neu: a review. Semin Oncol 1999; 26: 51-59.

8 Paterson MC et al. Correlation between c-erbB-2 amplification and risk of recurrent disease in node-negative breast cancer. Cancer Res 1991; 5155: 556-567.

9 Andrulis IL et al. Neu/erbB-2 amplification identifies a poor prognosis group of women with node-negative breast cancer. Toronto Breast Cancer Study Group. J Clin Oncol 1998; 16: 1340-1349.

10 Menard S, Tagliabue E, Campiglio M, Pupa S. Role of HER2 gene overexpression in breast carcinoma. J Cell Physiol 2000; 182: 150-162.

11 Ross JS, Fletcher JA. HER-2/neu (c-erb-B2) gene and protein in breast cancer. Am J Clin Pathol 1999; 112: S53-S67.

12 Muller WJ et al. Single-step induction of mammary adenocarcinoma in transgenic mice bearing the activated c-neu oncogene. Cell 1988; 54: 105-115.

13 Bouchard L, Lammare L, Tremblay PJ, Jolicoeur P. Stochastic appearance of mammary tumors in transgenic mice carrying the MMTV/c-neu oncogene. Cell 1989; 57: 931-936.

14 Guy CT, Cardiff RD, Muller WJ. Activated neu induces rapid tumor progression. J Biol Chem 1996; 271: 7673-7678.

$15 \mathrm{Yu} \mathrm{D}$ et al. c-erbB-2/neu overexpression enhances metastatic potential of human lung cancer cells by induction of metastasisassociated properties. Cancer Res 1994; 54: 3260-3266.

16 Yusa $\mathrm{K}$ et al. Low metastatic potential of clone from murine colon adenocarcinoma 26 increased by transfection of activated c-erbB-2 gene. J Natl Cancer Inst 1990; 82: 1633-1636.

17 Zhang L, Chang C-J, Bacus SS, Hung MC. Suppressed transformation and induced differention of HER-2/neu overexpressing breast cancer cells by emodin. Cancer Res 1995; 55: 38903896

18 Chang JY et al. The tumor suppression activity of E1A in HER2/neu overexpressing breast cancer. Oncogene 1997; 14: 561-568.

19 Suzuki $\mathrm{T}$ et al. Adenovirus-mediated ribozyme targeting of HER-2/neu inhibits in vivo growth of breast cancer cells. Gene Therapy 2000; 7: 241-248.

20 Zhang L, Hung MC. Sensitization of HER-2/neu-overexpressing non-small cell lung cancer cells to chemotherapeutic drugs by tyrosine kinase inhibitor emodin. Oncogene 1996; 12: 571-576.

21 Alroy I, Yarden Y. The ErbB signaling network in embryogenesis and oncogenesis: signal diversification through combinatorial ligand receptor interactions. FEBS Lett 1997; 410: 83-86.

22 Graus-Porta D, Beerli RR, Daly JM, Hynes NE. ErbB-2, the preferred heterodimerization partner of all ErbB receptors, is a mediator of lateral signaling. EMBO J 1997; 16: 1647-1655.

23 Messerle K, Schlegel J, Hynes N, Groner B. NIH/3T3 cells transformed with the activated erbB-2 oncogene can be phenotypically reverted by a kinase deficient dominant negative erbB2 variant. Mol Cell Endocrinol 1994; 105: 1-10.

24 Disis ML et al. Existent T-cell and antibody immunity to HER2/neu protein in patients with breast cancer. Cancer Res 1994; 54: 16-20.

25 Bei $\mathrm{R}$ et al. Immune responses to all erbB family receptors detectable in serum of cancer patients. Oncogene 1999; 18: 1267-1275.

26 Amici A et al. DNA vaccination with full-length or truncated Neu induces protective immunity against development of spontaneous mammary tumors in HER-2/neu transgenic mice. Gene Therapy 2000; 7: 703-706.

27 Bernards $\mathrm{R}$ et al. Effective tumor immunotherapy directed against an oncogene encoded product using a vaccinia virus vector. Proc Natl Acad Sci USA 1987; 84: 6854-6858.

28 Chen $\mathrm{Y}$ et al. Induction of erbB-2/neu specific protective and therapeutic antitumor immunity using genetically modified 
dendritic cells: enhanced efficacy by co-transduction of gene encoding IL-12. Gene Therapy 2001; 8: 316-323.

29 Deshane J et al. Targeted tumor killing via an intracellular antibody against erbB-2. J Clin Invest 1995; 96: 2980-2989.

30 Deshane $\mathrm{J}$ et al. Intracellular antibody against erbB-2 mediates targeted tumor cell eradication by apoptosis. Cancer Gene Ther 1996; 3: 89-98.

31 Xing $X$ et al. The ets protein PEA3 suppresses HER-2/neu overexpression and inhibits tumorigenesis. Nat Med 2000; 6: 189-195.

32 Ronchetti A et al. Immunogenicity of apoptotic cells in vivo: role of antigen load, antigen-presenting cells, and cytokines. J Immunol 1999; 163: 130-136.

33 Shi Y, Zheng W, Rock KL. Cell injury releases endogenous adjuvants that stimulate cytotoxic T cell responses. Proc Natl Acad Sci USA 2000; 97: 14590-14595.

34 Schreiber M, Muller WJ, Singh G, Graham FL. Comparison of

the effectiveness of adenovirus vectors expressing cyclin kinase inhibitors p16INK4A, p118INK4C, p19INK4D, p21(WAF1/CIP1) and p27KIP1 in inducing cell cycle arrest, apoptosis and inhibition of tumorigenicity. Oncogene 1999; 18: 1663-1676.

$35 \mathrm{Ng} \mathrm{P}$, Graham FL. Construction of first generation adenoviral vectors. In: Morgon JR (ed.). Methods in Molecular Medicine, Gene Therapy Protocols, 2nd edn. Humana Press: Totowa, 2001, pp 389-413.

36 Siegel PM, Dankort DL, Hardy WR, Muller WJ. Novel activating mutations in the neu proto-oncogene involved in induction of mammary tumors. Mol Cell Biol 1994; 14: 7068-7077.

37 Siegel PM, Muller WJ. Mutations affecting conserved cysteine residues within the extracellular domain of Neu promote receptor dimerization and activation. Proc Natl Acad Sci USA 1996; 93: $8878-8883$ 